\title{
OPTIMIZATION OF THE STABILIZATION SYSTEM FOR SUSPENSION IN ELECTROMAGNETIC ISOLATION DEVICES ACTIVE VIBRATION
}

Inna Plotnikova ${ }^{1, *}$, Maxim Vedyashkin ${ }^{1}$, Raisa Mustafina ${ }^{2}$, Igor Plotnikov ${ }^{3}$, Olga Tchaikovskaya ${ }^{4}$, Josefa $_{\text {Bastida }}{ }^{5}$, Mikhail Verpeta ${ }^{1}$

${ }^{1}$ Tomsk Polytechnic University, Institute of Non-Destructive Testing, 634050, Tomsk, Russia

${ }^{2}$ S.Toraighyrov Pavlodar State University, Department Electrical Engineering and Automation, 140000, Pavlodar, Kazakhstan

${ }^{3}$ Tomsk Polytechnic University, Institute of Power Engineering, 634050, Tomsk, Russia

${ }^{4}$ Tomsk State University, Faculty of Physics, 634050, Tomsk, Russia

${ }^{5}$ Universidad de Murcia, Department of Chemical Engineering, 30001, Murcia, Spain

\begin{abstract}
The research aims to study the vibration isolation device using the elastic forces of the electromagnetic suspension. The optimum gain values of the signal feedback circuit, which minimize the impact of noise introduced by the elements of the active vibration isolation device, are determined. The possibility of developing the vibration isolation device with the control circuit coefficients found on the boundary of the stability region is considered. The paper provides the amplitude-frequency characteristics of a single-mass electromagnetic suspension.
\end{abstract}

\section{Introduction}

The consideration of additional loads (static, vibration, seismic), which can lead to structural instability $[1,2]$ or even destruction [3-5], is relevant under the elevated static, mechanical and thermal loads. Nowadays, electromagnetic suspension systems are widely used in various fields of technology $[6,7]$.

It should be noted that both Russian [8, 9] and foreign researchers [10-12] have succeeded in stabilizing the electromagnetic suspension in active vibration protection devices. However, these results cannot be considered as a basis for the development of universal facilities capable of choosing the optimum structural solutions for vibration isolation devices with due regard to the functional area $[13,14]$. Further investigation of the optimization of the stabilization system for electromagnetic suspension is needed to obtain rational structural solutions of the object-oriented nature.

The research aims to determine the optimum parameters of the stabilization system under the law of change of the control voltage of the active vibration isolation device

*Corresponding author: inna@tpu.ru 
(AVID). With this, elastic forces of the electromagnetic suspension (EMS) are used to minimize the errors introduced by the elements of the vibration isolation device.

\section{Methodology}

In the general case, random disturbances affect the AVID that means that random signals will occur in sensors, voltage sources and electromagnets of the vibration isolation system [15]. A random process $X(t)$ is represented as $X(t)=m_{x}(t)+\stackrel{0}{X}(t)$, where $m_{x}(t)$ denotes the mathematical expectation (mean, systematic value) of a random process, and 0 $X(t)$ is a centered random processes. For example, the voltage converter generates the random signal $u(t)$, and this voltage can be represented as $u=U_{0}+\Delta u_{n}$, where $U_{0}$, reference voltage, is the system response to external disturbance and $\Delta u_{n}$, a random component (fluctuation) of the supply voltage, is the error introduced by the voltage source. Thus, the signals from other elements of the vibration isolation device can be represented in a similar way.

Therefore, we need to optimize the AVID supplied with EMS to reduce the impact of noise introduced by the elements of the vibration isolation device, i.e. to perform optimization in order to minimize the centered component of random signals. Variance is a characteristic of the centered random process

$$
D_{e x}=\sigma_{x}^{2}=M\left\{X(t)-m_{x}(t)\right\}^{2}=M\{\stackrel{0}{X}(t)\}^{2} .
$$

Variance is a measure of the scattering process $X(t)$ around the average value.

Take a minimum error variance introduced by the elements of the vibration isolation system as an optimization criterion. Determine the dependence of the selected criterion $D_{e x}$ on the stabilization system parameters $\alpha, \beta, \gamma$.

Due to the limitations imposed on the system parameters $\alpha, \beta, \gamma$ with regard to a specific area of the steady operation of the vibration isolation device, the AVID optimization is performed using the method of nonlinear programming. Conditions for the stable AVID operation are as follows: $\alpha>\frac{R b}{a}, \beta>\frac{b L_{0}-a^{2}}{a}, \gamma>\frac{-m R}{a}$, where $\mathrm{R}$ is the resistance of the EMS electromagnet winding; $L_{0}$ is the EMS inductance corresponding to the equilibrium point of the suspended body; $\mathrm{m}$ is the mass of the vibration-isolated object; $a$ is a linearization coefficient of the electromagnet traction, which depends on the electromagnet current and relative displacement of the object raised to the power $(-2) ; b$ is a linearization coefficient of the electromagnet traction, depending on the electromagnet current and relative displacement of the object raised to the power (-3) [16-18].

\section{Results and discussion}

\subsection{Choosing the active vibration isolation device parameters}

Perform the system optimization to reduce the noise introduced by the voltage converter into the AVID supplied with a single-mass EMS. Assume that the noise is a stationary random process such as noise with zero mean and constant spectral density $S_{u}=S_{0}$. In this case, variance of the control voltage fluctuations is determined by the formula: 


$$
D_{e u}=\int_{-\infty}^{\infty}\left|W_{u}(j \omega)\right|^{2} S_{u} d \omega=S_{0} \int_{-\infty}^{\infty}\left|W_{u}(j \omega)\right|^{2} d \omega=\min ,
$$

where $W_{u}$ is the closed-loop transfer function that relates the output value $\delta(t)$ to the noise introduced by the voltage regulator (Figure 1).

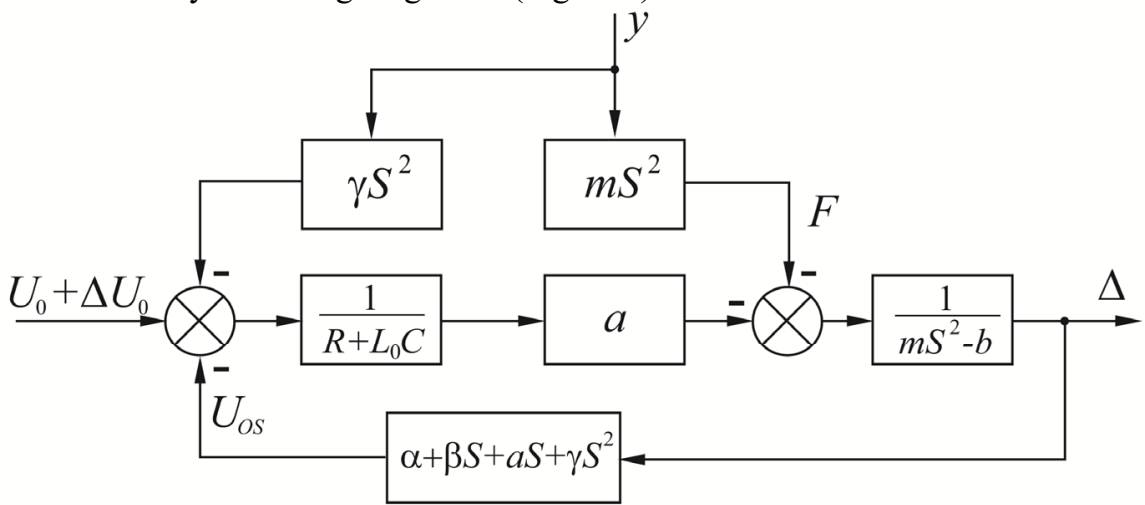

Figure 1. Block-diagram of the AVID supplied with a single-mass EMS.

$$
W_{u}(s)=\frac{-a}{m L_{0} S^{3}+(m R+a \gamma) S^{2}+\left(a^{2}+a \beta-b L_{0}\right) S+(a \alpha-R b)}
$$

Expression (2) with regard to the transfer function (3) takes the form

$$
D_{e u}=\int_{-\infty}^{\infty}\left|W_{u}(j \omega)\right|^{2} S_{u} d \omega=S_{0} \int_{-\infty}^{\infty}\left|W_{u}(j \omega)\right|^{2} d \omega=\min .
$$

Integrand (4) is a fractional rational function of the frequency $\omega$. Thus, the standard integral with the known solution is used to determine the error variance $D_{e u}$ [19].

The standard integral for this case is written as

$$
I_{3}=\frac{1}{2 \pi j} \int_{-\infty}^{\infty} \frac{B_{3}(\omega)}{A_{3}(\omega) A_{3}(-\omega)} d \omega,
$$

where $A_{3}(\omega)=a_{0} \omega^{3}+a_{1} \omega^{2}+a_{2} \omega+a_{3} ; B_{3}(\omega)=b_{0} \omega^{4}+b_{1} \omega^{2}+b_{2}$.

The solution of the standard integral $\mathrm{I}_{3}$ can be obtained from the expression

$$
I_{3}=\frac{-a_{2} b_{0}+a_{0} b_{1}-\frac{a_{0} a_{1} b_{2}}{a_{3}}}{2 a_{0}\left(a_{0} a_{3}-a_{1} a_{2}\right)}
$$

in the considered case $a_{0}=-j L_{0} m ; a_{1}=-\left(R_{m}+a \gamma\right) ; a_{2}=j\left(a^{2}+a \beta-b L_{0}\right)$; $a_{3}=(a \alpha-R b) ; b_{0}=0 ; b_{1}=0 ; b_{2}=a^{2}$.

The desired expression for the error variance with regard to expressions (5) and (6) can be represented as

$$
D_{e u}=\frac{\pi a^{2} S_{o u}(m R+a \gamma)}{(a \alpha-R b)\left[(m R+a \gamma)\left(a^{2}+a \beta-b L_{0}\right)-\left(L_{0} m(a \alpha-R b)\right)\right]} .
$$


Following the procedure of nonlinear programming, write Lagrangian function with regard to the limitations imposed on the system parameters

$$
F=D_{e u}(\alpha ; \beta ; \gamma)+\lambda_{1}\left(\frac{R b}{a}-\alpha\right)+\lambda_{2}\left(\frac{b L_{0}-a^{2}}{a}-\beta\right)+\lambda_{3}\left(\frac{-m R}{a}-\gamma\right),
$$

where $\lambda_{i}(i=1,2,3)$ is Lagrangian multiplier.

When using the method of nonlinear programming, the optimum values of the coefficients $\alpha^{*}, \beta^{*}$, and $\gamma^{*}$ are determined from the system of equations:

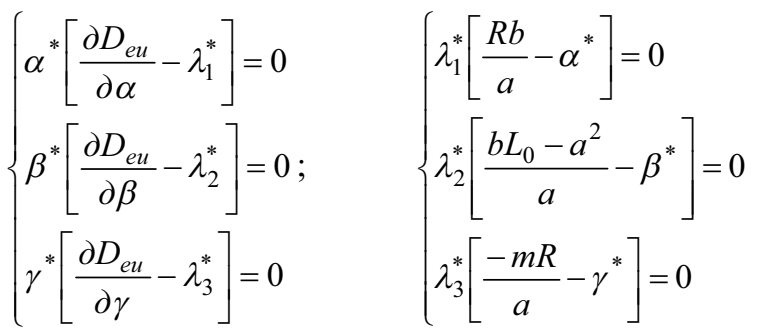

Among the possible solutions of system (9), choose those that meet the conditions of existence of the extreme point of the function considered [1]. Thus, we obtain $\lambda_{1}^{*}=\lambda_{2}^{*}=\lambda_{3}^{*}=0$, and the optimum gain values are calculated from the relations:

$$
\frac{\partial D_{e u}}{\partial \alpha}=0 ; \frac{\partial D_{e u}}{\partial \beta}=0 ; \frac{\partial D_{e u}}{\partial \gamma}=0 .
$$

Substituting relation (7) obtained for the variance into expression (10), we find the system of equations to determine the optimum values of the gains $\alpha, \beta$, and $\gamma$, which ensure the fulfillment of condition (2):

$$
\left\{\begin{array}{c}
-\pi a^{2} S_{0}(m R+a \gamma)\left[a(m R+a \gamma)\left(a^{2}+a \beta-b L_{0}\right)-2 L_{0} m a(a \alpha-b R)\right]=0 \\
-\pi a^{2} S_{0}(m R+a \gamma)[a(m R+a \gamma)(a \alpha-b R)]=0 \\
S_{0} \pi a^{3}(a \alpha-b R)\left\{\left[(m R+a \gamma)\left(a^{2}+a \beta-b L_{0}\right)-L_{0} m(a \alpha-b R)\right]-\right. \\
\left.-S_{0} \pi a^{3}(m R+a \gamma)\left(a^{2}+a \beta-b L_{0}\right)\right\}=0
\end{array}\right.
$$

The optimum gain values obtained on the border of the system stability follow from the joint solution of equations (11)

$$
\alpha^{*}=\frac{R b}{a} ; \beta^{*}=\frac{b L_{0}-a^{2}}{a} ; \gamma^{*}=-\frac{m R}{a} .
$$

Therefore, we take the gain values based on the conditions of stable operation of the vibration isolation device.

Perform optimization of the considered AVID supplied with EMS to reduce the errors introduced by the position, velocity and acceleration sensors.

The optimization criterion is written similarly to (2)

$$
D_{e d}=\int_{-\infty}^{\infty}\left|W_{d}(j \omega)\right|^{2} S_{d} d \omega=\min ,
$$

where $W_{d}(p)$ is the transfer function that relates the output value $\Delta(\mathrm{t})$ to the noise introduced by the position sensors 


$$
W_{d}(s)=\frac{a \alpha+a(a+\beta) S+a \gamma S^{2}}{m L_{0} S^{3}+(m R+a \gamma) S^{2}+\left(a^{2}+a \beta-b L_{0}\right) S+(a \alpha-R b)} .
$$

Assume that the considered noise is a stationary random process such as white noise with zero mean and constant spectral density $S_{d}(\omega)=S_{d 0}=$ const. Then expression (13) with regard to the conditions and formula (14) is written as:

$$
D_{e d}=S_{d 0} \int_{-\infty}^{\infty} \frac{\left|a \alpha-\omega^{2} a \gamma+j \omega a(a+\beta)\right|^{2}}{\left|-j \omega^{3} L_{0} m-\omega^{2}(m R+a \gamma)+j \omega\left(a^{2}+a \beta-b L_{0}\right)+(a \alpha-R b)\right|^{2}} d \omega .
$$

Using the solution of the standard integral $I_{3}(\omega)$, we find that

$$
D_{e d}=\pi S_{d 0} \frac{(a \alpha-R b)\left[a^{2} \gamma^{2}\left(a^{2}+a \beta-b L_{0}\right)+L_{0} m\left(a^{2}(a+\beta)^{2}-2 a^{2} \alpha \gamma\right]\right.}{L_{0} m\left[(m R+a \gamma)\left(a^{2}+a \beta-b L_{0}\right)-L_{0} m(a \alpha-R b)\right](a \alpha-R b)} .
$$

\subsection{Optimum values of amplification coefficient}

A comparison of the characteristics of Figures 2 and 3 reveals that the acceleration sensor installation onto the vibration-isolated object and introduction of the additional relation to the acceleration output value reduce the initial frequency value, which marks the beginning of the effective vibration isolation of the object, by several hertz.

With regard to the imposed limitations, the optimum gain values $\alpha^{*}, \beta^{*}$ and $\gamma^{*}$, reducing the effect of the sensor errors on the output signal circuit $\delta(t)$, are determined similarly to that of the error reduction from fluctuations in the supply voltage.

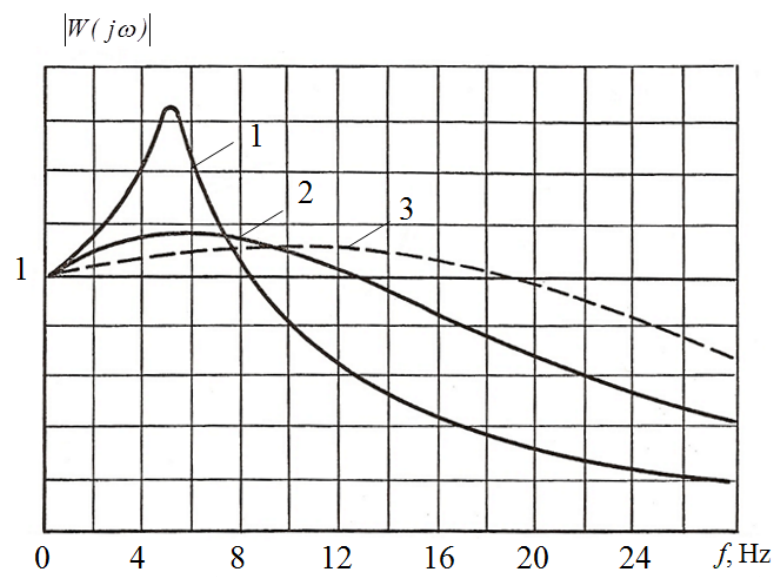

Figure 2. Amplitude-frequency characteristics (transfer coefficients) of the single-mass EMS $(\alpha=$ const $)$. 


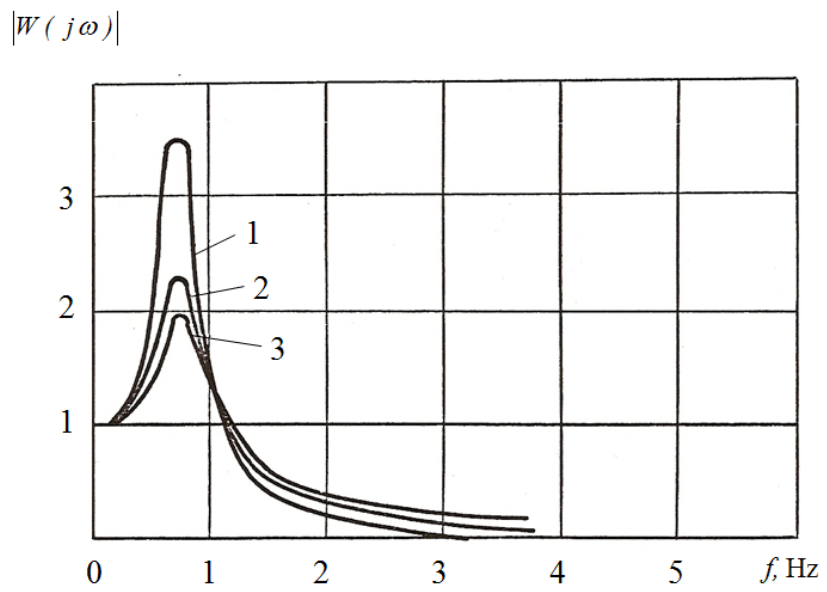

Figure 3. Amplitude-frequency characteristics of the EMS with the additional relation to acceleration of the inertial element ( $\alpha-$ const $)$.

\section{Conclusion}

The optimum gain values of the signal feedback circuit, which minimize the impact of noise introduced by the elements of the active vibration isolation device, are obtained on the boundary of stability. The vibration isolation devices, which control circuit coefficients are found on the boundary of the stability region, can be developed during the variable structure system design. Due to the fact that the system development implies a number of difficulties, the values of the feedback loop coefficients are to be selected on the basis of stable operation of the vibration isolation device and be close to the optimum values to design the vibration isolation devices with fixed structure. In this case, a suboptimal device is developed, but not the entirely optimum device of the active vibration isolation.

\section{References}

[1] A.P. Surzhikov, O.V. Galtseva, E.A. Vasendina, V.A. Vlasov, E.V. Nikolaev, IOP Conf. Ser.: Mater. Sci. Eng. 110, 012002 (2016) doi : 10.1088/1757$899 X / 110 / 1 / 012002$

[2] E.N. Lysenko, A.P. Surzhikov, S.P. Zhuravkov, V.A. Vlasov, A.V. Pustovalov, N.A. Yavorovsky, J. Therm. Anal. Calorim. 115(2), 1447 (2014) doi: 10.1007/s10973-013-3456-x

[3] Y.A. Chursin, E.M. Fedorov, Optics\&Laser Technology 67, 86 (2015) doi: 10.1016/j.optlastec.2014.09.017

[4] M.V. Vedyashkin, O.P. Muravlev, Russ. Electrical Eng. 84(7), 397 (2013) doi: 10.3103/S1068371213070146

[5] O.V. Galtseva, S.V. Bordunov, S.N. Torgaev, IOP Conf. Ser.: Mater. Sci. Eng. 110, 012094 (2016) doi: 10.1088/1757-899X/110/1/012094

[6] R.M. Mustafina, I.A. Plotnikov, I.V. Plotnikova, O.N. Tchaikovskaya, J. Phys: Conf. Ser. 671, 012046 (2016) doi: 10.1088/1742-6596/671/1/012046

[7] O.V. Galtseva, G.V. Vavilova, I.V. Plotnikova, SENSIG '15, 91 (2015)

[8] I.J. Bezbah, V.A. Melik-Shahnazarov, D.V. Fiyanchuk, V.I. Strelov, SCIENCE\&EDUCATION 9, 317 (2012) 
[9] V. A. Melik-Shakhnazarov, V. I. Strelov, D. V. Sofiyanchuk, I. Zh. Bezbakh, Techn. Phys. Lett. 38 (3), 283 (2012) doi: 10.1134/S1063785012030303

[10] N. Docquier, A. Poncelet, M. Delannoy, P. Fisette, V. Sys. Dyn. 48, 1439 (2010)

[11] S.V. Bordunov, O.V. Galtseva, J. Phys: Conf. Ser. 671, 012009 (2016) doi:10.1088/1742-6596/671/1/012009

[12] M. Jamil , A.A. Janjua, I. Rafique, S.I. Butt, Y. Ayaz, S.O. Gilani, Life Sc. J. 10 (SPLISSUE 12), 653 (2013)

[13] K. Kitching, D.J. Cole, D. Cebon, Journal of Dynamic Systems, Measurement and Control, Transactions of the ASME 122,498 (2000)

[14] J. Lei, Z. Jiang, Y.-L. Li, W.-X. Li, Int. J. Cont. 87(10), 2081 (2014) doi:10.1080/00207179.2014.901564

[15] I. Mihai, F. Andronic, Mech. 20, 64 (2014) doi: 10.5755/j01.mech.20.1.6591

[16] K. Frolov, A. Furman, Applied theory of vibration protection systems (Mashinostroenie, Moscow, 1980)

[17] R. I. Furundjiev, Designing of optimal vibration isolation systems (Visshaya shkola, Minsk, 1971)

[18] X.Wen, C. Zhu, R. Hu, Ch. Song, J. Zhang, MATEC Web of Conferences 35, 02010 (2015) doi: 10.1051/matecconf $/ 20153502010$

[19] M.F. Rosin, V.S. Buligin, Statistical dynamics and efficiency systems theory (Mashinostroenie, Moscow, 1981) 